\title{
Coulisses
}

Revue de théâtre

6 | Printemps 1992

Varia

\section{Le théâtre en Thaïlande}

\section{Olivier Maret}

\section{OpenEdition}

Journals

Édition électronique

URL : http://journals.openedition.org/coulisses/2033

DOI : $10.4000 /$ coulisses.2033

ISSN : 2546-9460

\section{Éditeur}

Presses universitaires de Franche-Comté

\section{Édition imprimée}

Date de publication : 1 juin 1992

ISSN : 1150-594X

\section{Référence électronique}

Olivier Maret, "Le théâtre en Thaillande », Coulisses [En ligne], 6 | Printemps 1992, mis en ligne le 15 mars 2019, consulté le 28 octobre 2019. URL : http://journals.openedition.org/coulisses/2033 ; DOI : $10.4000 /$ coulisses. 2033

Ce document a été généré automatiquement le 28 octobre 2019

Coulisses 


\title{
Le théâtre en Thaillande
}

\author{
Olivier Maret
}

1 La Thaillande s'enorgueillit à juste titre d'une tradition théâtrale propre, et de haute réputation. L'imagerie touristique met en bonne place, parmi les fruits exotiques et les temples bouddhistes, ces charmantes danseuses aux costumes miroitants et aux gestes raffinés, qui exécutent des danses autrefois réservées aux seuls plaisirs royaux.

2 Pourtant, arrivé au "Pays du sourire », et après quelques recherches infructueuses, c'est la rareté des spectacles qui frappe. Aucun théâtre permanent n'existe dans les grandes villes des provinces, et les spectacles itinérants sont peu nombreux et peu annoncés. A Bangkok seulement il existe un bâtiment construit spécialement pour les représentations de théâtre classique : le Théâtre Silpakorn. Mais son fonctionnement revient cher, et les représentations n'y ont lieu que les samedis et dimanches de certaines semaines. Si l'on ajoute que chaque ville - voire chaque village - possède une ou plusieurs salles de cinéma, et que dans chaque foyer fonctionne à peu près en permanence un poste de télévision, on a bien vite compris que, s'il fait partie de la culture thaïe, par son originalité le théâtre ne touche qu'une part infime des Thaïlandais, et qu'il se trouve menacé par la concurrence des moyens de communication nés des technologies occidentales. Cependant, la remarquable continuité de la monarchie thaïe, et l'action de plusieurs personnalités cultivées, ont permis de préserver les formes anciennes du théâtre, malgré les transformations colossales survenues dans le pays depuis le début du siècle. Discret dans la trépidante vie thaillandaise, ce théâtre vaut que l'on aille à sa découverte, et que l'on prenne le temps de plier son esprit à ses contours singuliers.

3 Comme l'ensemble des théâtres asiatiques, le théâtre thaïlandais a pour caractéristique de mêler ce que nous séparons en genres bien distincts. Le spectacle est bien la représentation d'un récit par des acteurs, mais les actions sont plus dansées que jouées, sans souci d'imiter le réel. De plus, qu'il soit une narration ou un dialogue, le texte est dit par des récitants, placés sur le côté de la scène. Enfin, paroles et actions sont accompagnées par un petit orchestre, lui aussi visible sur la scène. Chant, musique, danse et jeu d'acteurs se fondent donc, et c'est l'harmonie et l'équilibre de ces différentes composantes qui donnent toute sa valeur au spectacle. 
4 Nous présenterons quatre genres dramatiques traditionnels, sans prétendre à l'exhaustivité, les genres se divisant en sous-genres qui varient selon les époques et les régions. Du fait de sa quasi-disparition, nous n'avons eu accès au Théâtre d'ombre, le Nang Yay, que par la vidéo, les photographies et les témoignages écrits. Nous avons pu en revanche assister aux représentations des anciens genres de cour, le Lakhon et le Khon, et du genre populaire encore bien répandu, le Liké.

5 L'art théâtral le plus prestigieux et le plus fascinant est sans aucun doute le Lakhon, Drame-dansé accompagné de récitation et de musique. Joué à l'origine uniquement par des femmes attachées à la personne du roi, cet art est le lointain descendant des danses indiennes exécutées pour le culte des divinités hindoues. L'orchestre, ou Pi Phat, placé à gauche de la scène, est composé de plusieurs xylophones, d'une flûte, de gongs, de violons thaïs et de tambours. A côté des musiciens, les cinq ou six récitants scandent le texte devant un micro, en chœur ou séparément. Tous sont assis à même le sol, et suivent attentivement le déroulement de l'action. Les actrices-danseuses, enfin, n'ayant à de rares exceptions près aucun texte à dire, développent un langage gestuel complexe, dont chaque figure correspond à un sens bien déterminé, codifié dans un alphabet. Aucune tension dramatique n'intervient dans ces évolutions, mais une grâce heureuse s'en dégage, accentuée par les costumes miroitants et les coiffes allongées des danseuses. Le récit le plus souvent représenté est celui des aventures d'Inao, prince chevaleresque en quête de sa bien-aimée Busada, chaque épisode de la légende constituant un spectacle en lui-même. Les représentations ont lieu l'après-midi, au Théâtre National Silpakorn, dans une atmosphère sans protocole qui autorise les musiciens à parler et rire entre eux et les spectateurs à bavarder et manger pendant le spectacle.

Danseuse de Lakhon

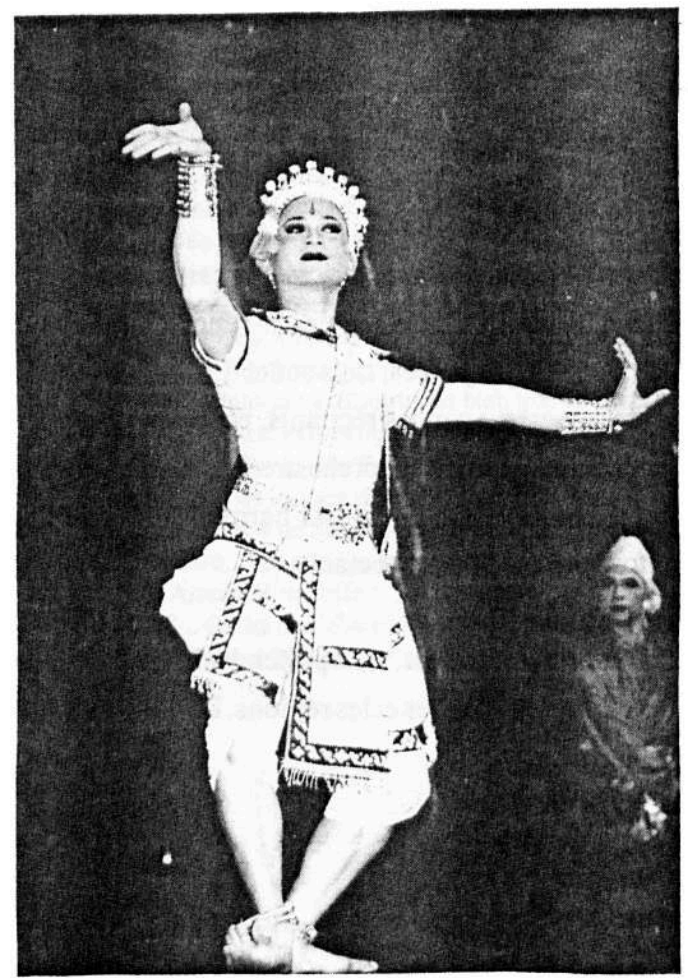

Coulisses, 6 | Printemps 1992 
6 La seconde forme de divertissement royal est le Théâtre d'ombre, ou Nang Yay. Nang signifie "cuir ", les figures représentées derrière l'écran lumineux étant faites de peaux de buffle; Yay veut dire "grand", par opposition avec les petites figures articulées d'origine malaise. Ces marionnettes de cuir, pouvant atteindre $\operatorname{lm} 50$ de haut, sont découpées et évidées avec un extrême souci du détail, chacune représentant un ou deux personnages, ainsi que des éléments de décor. Elles sont animées par des manipulateurs-danseurs qui les tiennent au-dessus de leur tête par deux manches en bambou. Les manipulateurs, visibles dans la transparence de l'écran, évoluent par mouvements latéraux, accompagnés par l'orchestre et les récitants. Ils passent parfois devant l'écran, ce qui explique que certaines figures de cuir soient colorées.

7 C'est par développement de ce jeu, sous le regard direct des spectateurs, que le Théâtre d'ombre a donné naissance à une autre forme de divertissement royal, le Khon ou Drame-masqué. En effet, on retrouve dans les mouvements des acteurs du Khon les déplacements latéraux des marionnettistes du Nang. De plus, le Khon a en commun avec le Nang de tirer ses thèmes narratifs du Ramakien, version thaïe de l'épopée indienne Ramayana. Le Ramakien, thème pictural et littéraire très populaire, met en scène trois groupes de personnages : les Humains, qui ont pour chef Rama, incarnation de Vishnu; l'armée des Singes, commandée par Hanuman; les Démons (ou Géants) dirigés par Tosakanth. Suite à l'enlèvement par Tosakanth de Sita, la femme de Rama, les Humains alliés aux Singes, affrontent les Démons, dans une suite de combats singuliers ou collectifs, jusqu'à la victoire finale. Les Singes et les Démons sont des personnages masqués, contrairement aux Humains, dont le costume est semblable à celui des danseuses du Lakhon. Les masques aux physionomies saisissantes que l'on retrouve dans l'ornementation des temples de style khmer, englobent entièrement la tête des comédiens et permettent d'identifier les personnages parmi les dizaines que comporte le Ramakien. A l'exception du personnage de Sita, le Khon est joué exclusivement par des hommes, et les récitants sont eux aussi tous masculins. Outre les scènes de palais où s'ourdissent les assauts contre la capitale ennemie, les mouvements des comédiens culminent dans de spectaculaires affrontements où la coopération acrobatique entre les combattants l'emporte sur la violence. Les Singes en particulier suscitent l'admiration par leur adresse corporelle et leurs mouvements incessants même au repos : ils s'agitent, se grattent, attrapent des mouches, font signe au public, et donnent ainsi un air de comédie burlesque à ce combat entre le Bien et le Mal. 


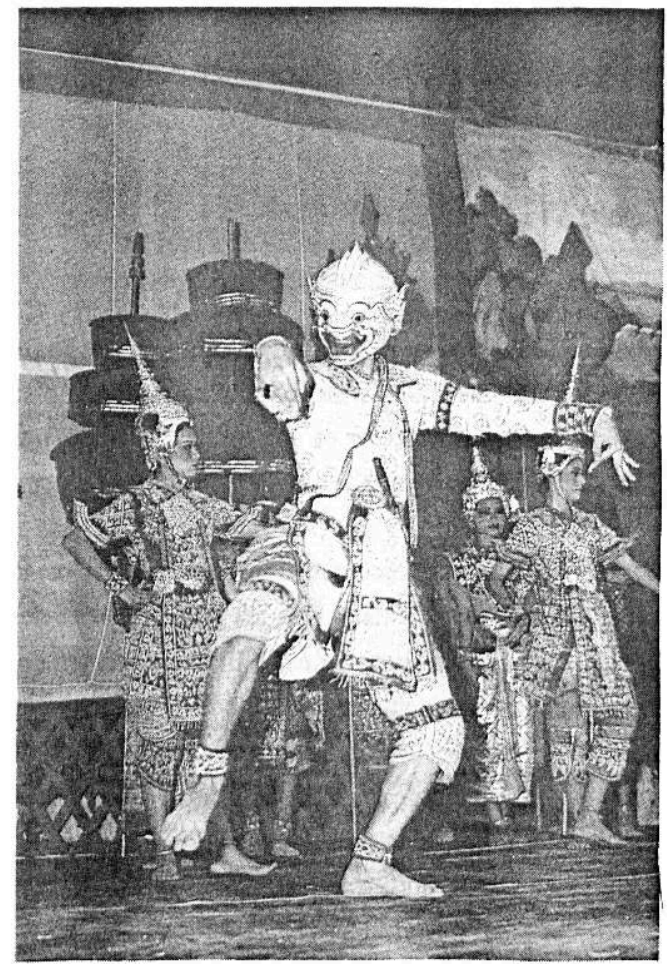

8 La principale forme de théâtre populaire est le Liké, également appelé "Opéra populaire" car les acteurs disent eux-mêmes les répliques de leur personnage en les improvisant en partie. Le nom de Liké vient vraisemblablement du mot malais dikay qui désigne un chant musulman shiite invoquant la bénédiction d'Allah sur le roi. Ce chant fut repris au XIXème siècle par des troupes itinérantes pour servir d'introduction à leur spectacle et rassembler le public. Les représentations ont lieu dans la cour des temples bouddhiques et sont offertes par un particulier à l'occasion d'une fête religieuse ou d'une crémation. On peut également voir le Liké dans certaines foires, financé cette fois par une municipalité. Le jeu est une adaptation populaire, d'un faible niveau, du théâtre classique. On y retrouve les intrigues princières, les costumes miroitants, quelques esquisses de danses et un orchestre réduit à trois musiciens qui rythme les dialogues. L'intérêt du Liké réside dans la vivacité des répliques et dans les jeux de mots souvent improvisés dont le sens échappe presque totalement à un étranger. Mais c'est l'impression de pauvreté tant artistique que sociale qui domine. La scène est souvent une construction rudimentaire où les coulisses sont délimitées par des tissus tendus. Des néons éclairent un décor peint, aux couleurs criardes, figurant un jardin ou la salle d'un palais. De part et d'autre, de grands haut-parleurs amplifient à l'extrême la voix aigre des comédiens. Ceux-ci sont donc, pendant leur jeu, sans cesse contraints d'échanger un microphone. Originellement, les acteurs du Liké avaient étudié avec les acteurs classiques. Mais il semble qu'aujourd'hui ils n'aient à leur actif qu'une certaine pratique de l'improvisation, et le désir d'une vie un peu moins misérable que celle de leur milieu d'origine. Le public est à l'image de la troupe : ce sont les plus pauvres qui viennent s'asseoir à même le sol, devant la scène, pour suivre la nuit entière les aventures de princes d'un autre monde. 
9 La Thaïlande a su conserver de façon remarquable son théâtre traditionnel. Mais peuton aller jusqu'à parler d'un théâtre vivant et créatif? Très ouverte à l'Occident, la Thaïlande suit depuis le début du siècle une politique d'adaptation permanente aux techniques modernes et à l'économie de marché, ce qui lui a permis d'atteindre une prospérité relative, en comparaison avec ses voisins. Mais cette évolution empruntée de l'étranger met en péril les structures et les moyens d'expression de la culture thaïe, élaborés autour de représentations évidemment très différentes de celles qui prévalent dans la Thaillande contemporaine. Cette menace pèse tout particulièrement sur les arts dramatiques, et amène trois conséquences : la disparition de certains genres comme le Théâtre d'ombre, nous l'avons dit; la reprise pure et simple du répertoire et de la dramaturgie de l'Occident, exclusivement pour l'élite cultivée; ou enfin l'attachement à une forme académique qui caractérise les productions du Théâtre National, administré par l'Etat. Entre ces trois voies, y a-t-il la possibilité d'une renaissance de la création à partir des formes du théâtre classique? Le théâtre peut-il proposer aux Thaïlandais les images d'une réconciliation entre leurs traditions et leur mode de vie réel ? La réponse dépend en grande partie de l'évolution de la société thaïe dans son ensemble et des solutions qu'elle trouvera aux contradictions qui la traversent 